\title{
Manfred Hettling, Richard Pohle (dir.), Bürgertum. Bilanzen, Perspektiven, Begriffe
}

\section{Antonin Dubois}

\section{OpenEdition}

\section{Journals}

Édition électronique

URL : http://journals.openedition.org/ifha/10407

DOI : $10.4000 /$ ifha. 10407

ISSN : 2198-8943

\section{Éditeur}

IFRA - Institut franco-allemand (sciences historiques et sociales)

Référence électronique

Antonin Dubois, « Manfred Hettling, Richard Pohle (dir.), Bürgertum. Bilanzen, Perspektiven, Begriffe », Revue de l'IFHA [En ligne], Date de recension, mis en ligne le 26 octobre 2019, consulté le 24 septembre 2020. URL : http://journals.openedition.org/ifha/10407 ; DOI : https://doi.org/10.4000/ifha.10407

Ce document a été généré automatiquement le 24 septembre 2020.

(CIFHA 


\section{Manfred Hettling, Richard Pohle (dir.), Bürgertum. Bilanzen, Perspektiven, Begriffe}

Antonin Dubois

\section{RÉFÉRENCE}

Manfred Hettling, Richard Pohle (dir.), Bürgertum. Bilanzen, Perspektiven, Begriffe, Göttingen, Vandenhoeck \& Ruprecht, coll. « Bürgertum Neue Folge », n 18, 2019, 489 p., $59,99 €$ 
La bourgeoisie, ou plutôt faudrait-il dire les bourgeoisies, constituent depuis près d'un demi-siècle l'un des objets de recherche les plus prisés de l'histoire contemporaine allemande. Le «siècle bourgeois » qu'est le XIX ${ }^{e}$ siècle a été la période privilégiée d'innombrables études et de plusieurs projets collectifs. Ces travaux sur la bourgeoisie ont été parmi les pièces maîtresses du fameux débat sur le Sonderweg, sur le chemin particulier qu'aurait emprunté l'Allemagne par rapport à ses voisins ouest-européens et d'outre-Atlantique et qui expliquerait l'échec de la démocratie et le régime nazi. Cet ouvrage dirigé par Manfred Hettling et Richard Pohle de l'université de Halle entend non pas revenir sur le potentiel "déficit bourgeois" («Defizit an

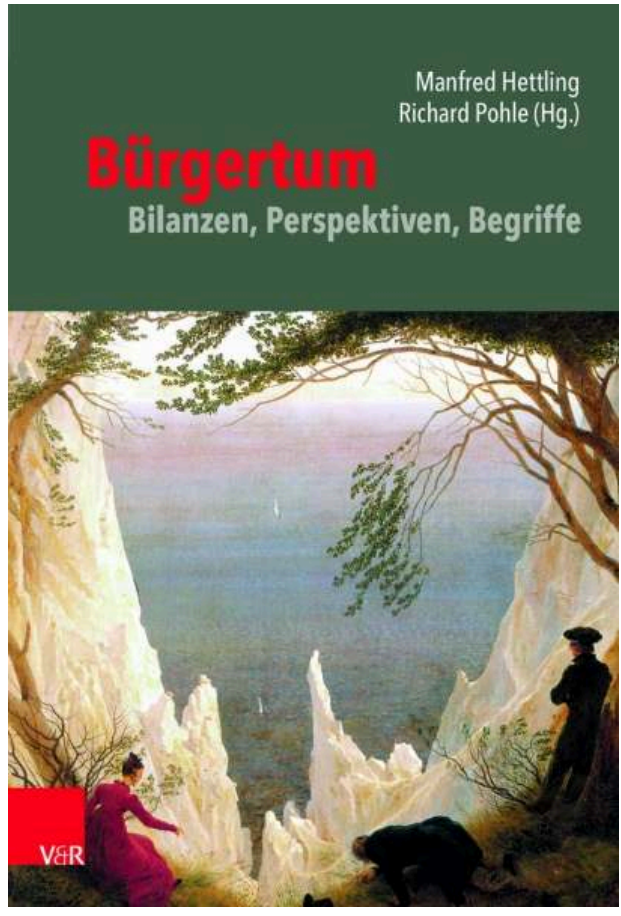
Bürgerlichkeit »), sur l'absence duquel tout le monde s'entend désormais, mais faire le bilan de ces décennies de recherche et proposer des pistes à explorer sur les sujets qui n'ont encore que peu intéressé les historiens et historiennes.

En introduction, Manfred Hettling remet brièvement en perspective l'histoire de la recherche sur la bourgeoisie en Allemagne et ses grands principes (sémantique, chronologie, questionnements, approches, débats), puis insiste surtout sur ce qu'il considère comme un manque qu'il s'est lui-même efforcé de combler progressivement avec ses propres travaux et à laquelle une section de l'ouvrage est consacrée : l'histoire de la bourgeoisie au $\mathrm{XX}^{\mathrm{e}}$ siècle.

Les premières esquisses par M. Hettling d'un retour sur l'historiographie allemande de la bourgeoisie sont développées dans la première partie, qui dresse des bilans de projets et de groupes de recherche qui se sont consacrés entre la fin des années 1970 et des années 1990 à l'étude de l'histoire de la bourgeoisie en Allemagne: Dieter Langewiesche se penche sur l'Arbeitskreis für moderne Sozialgeschichte, Dieter Hein sur le projet mené sur la Stadtbürgertum à Francfort-sur-le-Main sous l'égide de Lothar Gall, et Thomas Mergel sur le projet dirigé par Jürgen Kocka à Bielefeld. Ces trois articles sont particulièrement riches d'informations quant à l'historiographie allemande des dernières décennies $\mathrm{du} \mathrm{XX}^{\mathrm{e}}$ siècle. Les acteurs, les débats et les thématiques ayant circulé entre ces trois groupes et ces projets, très bien résumés et présentés, se recoupent assez largement. Cependant les méthodes suivies ne sont pas toujours les mêmes. Le projet francfortois, par exemple, se caractérise par une grande cohésion : selon une typologie préalablement établie de six types de ville, seize villes ont été choisies sur lesquelles des recherches monographiques approfondies ont été menées. Bien financés, ces vastes projets ont permis une productivité impressionnante. Ainsi, Thomas Mergel rappelle que dans l'ouvrage faisant le bilan définitif du projet de Bielefeld (1986-1997) sont recensées 561 publications, 31 thèses de doctorat et 15 thèses d'habilitation permises grâce à ce projet. De tels bilans, qui reviennent de manière 
détachée et parfois critique sur de telles entreprises de recherche, sont rarement publiés. Ils sont pourtant particulièrement utiles tant à la recherche qu'à l'enseignement, mais aussi à la connaissance par les historiens de l'histoire de leur discipline. Ils participent donc ici grandement à l'originalité et à l'intérêt de l'ouvrage. L'absence d'un bilan équivalent sur les recherches menées depuis vingt ans est de ce point de vue étonnante et l'on doit se contenter d'indications partielles, alors que plusieurs thématiques auraient pu s'y prêter - comme l'histoire des femmes, nous y reviendrons.

Les parties II et III sont bien plus classiques dans leur forme : des recherches actuelles sur la bourgeoisie aux XIX et (surtout) $\mathrm{XX}^{\mathrm{e}}$ siècles, qui permettent d'approfondir et de combler un certain nombre de manques des travaux menés au cours des années 1980-1990 et dont les acquis viennent d'être résumés. Les neuf articles qui composent ces deux parties embrassent des sujets extrêmement différents, emploient des méthodes tout aussi diverses et leur qualité varie d'un texte à l'autre, comme c'est souvent le cas avec ce genre d'ouvrage. Il faut surtout noter que les thématiques choisies ne trouvent sens que par rapport aux débats et aux manques qui caractérisent les travaux pionniers présentés dans la première partie. Pour le XIX siècle, les recherches anciennes de Rudolf Vierhaus sur les « éduqués » (Gebildeten) et la définition culturaliste de la bourgeoisie par M. Rainer Lespius comme Vergesellschaftung, inspirée par Max Weber, trouvent directement écho dans les articles de Reinhard Blänkner sur les Gebildeten et de Hartmann Tyrell sur le concept de Lebensführung (style de vie) chez Weber. Thomas Mergel insiste dans son bilan sur l'intérêt quasi-nul porté par les historiens du projet de Bielefeld pour la petite bourgeoisie, l'article de Richard Pohle porte à l'inverse sur les aspirants pasteurs qui doivent se contenter d'une position de précepteurs. Pour ne citer que deux des articles sur le $\mathrm{XX}^{\mathrm{e}}$ siècle, celui de Werner Plumpe revient sur la thématique classique de la «crise » de la bourgeoisie, ici durant la Première Guerre mondiale, en revenant au plus proche des biographies (carrières, conditions de vie, expériences de la guerre), tandis que celui de Philip Wagner traite de l'apprentissage du jeu démocratique dans la RFA au lendemain de la Seconde Guerre mondiale, montrant à sa manière que même après l'expérience nationale-socialiste, valeurs bourgeoises et démocratie ne sont pas antinomiques. Du fait de l'effondrement économique de l'Allemagne après la Grande Guerre, plusieurs auteurs font une part plus importante aux questions économiques que ceux s'intéressant au XIX siècle. Mais les auteurs des deux parties se rejoignent sur un point fondamental. Ils montrent (ainsi que les deux éditeurs qui ont fait le choix de sélectionner ces textes) l'importance durable des problématiques et des questionnements des recherches pionnières. Plus de deux décennies après la fin annoncée du Sonderweg, les recherches actuelles restent, au moins à un certain degré et bien que cela ne soit pas toujours ouvertement accepté, déterminées par ces débats qui ont si fortement agité l'historiographie et la société allemandes durant la seconde moitié du siècle dernier.

La partie IV fait elle aussi écho à certaines des recherches parmi les plus anciennes sur la bourgeoisie, celles d'une histoire sémantique des termes Bürger ou Bürgertum, par exemple celles de Reinhart Koselleck. Elles apportent cependant d'importantes nouveautés dans la mesure où elles sortent du cadre européocentré voire germanocentré : Reinhard Schulze traite des concepts d'autodéfinition bourgeoise dans les sociétés du Proche-Orient, Thomas Fröhlich et Tino Schölz reviennent sur l'histoire sémantique du terme ou des termes équivalents à Bürger respectivement en Chine et au Japon. Là aussi, les débats et les méthodes de l'historiographie allemande, notamment 
la Begriffsgeschichte de Koselleck et son concept de Sattelzeit (époque charnière) sont pleinement intégrés, bien que réadaptés, dans ces travaux sur des aires extraeuropéennes.

C'est de l'histoire globale que se réclame Christof Dejung dans sa conclusion, intitulée «Bourgeois du monde. Réflexions sur une histoire globale des classes moyennes durant le long XIX ${ }^{e}$ siècle ». Ce texte pose un problème sémantique majeur : sous la plume de C. Dejung, la terminologie de la bourgeoisie (Bürgertum) qui est utilisée pour les Européens est remplacée par celle de "classes moyennes» (Mittelklassen, toujours utilisé au pluriel) en ce qui concerne les non-Européens, afin, dit-il, de ne pas appliquer le vocabulaire des dominants aux dominés. Outre les problèmes intrinsèques à l'utilisation du terme "classes moyennes", sur laquelle de nombreux historiens et sociologues sont déjà revenus, cette mise en équivalence de la bourgeoisie à un groupe socio-économique (alors que les autres auteurs de l'ouvrage adoptent une définition plutôt culturelle, quasi-hégémonique dans la littérature allemande depuis Lepsius) n'est pas injustifiée en soi, mais n'est ni explicitée ni argumentée. C. Dejung va même jusqu'à indiquer que l'historiographie contemporaine traite les classes moyennes comme des « couches sociales extrêmement hétérogènes " (« äußerst heterogene soziale Schichten ", p. 475-476), témoignant de l'absence de toute définition de cette expression. Cette conclusion très générale, qui porte sur de grandes tendances et ne s'appuie que sur la littérature secondaire sans en faire un bilan systématique, tranche avec les études souvent resserrées qui l'ont précédée et en semble assez largement déconnectée.

Une critique majeure plus générale peut être apportée à l'entreprise menée par M. Hettling et R. Pohle. Il a fallu, à la toute fin des années 1980, que des historiennes s'attachent à étudier la place des femmes dans la bourgeoisie en général (Ute Frevert) ou dans les métiers bourgeois (Claudia Huerkamp), pour que l'histoire allemande de la bourgeoisie cesse d'être une histoire entièrement masculine. Il est regrettable que dans cet imposant ouvrage collectif, un seul chapitre sur dix-sept soit le fait d'une historienne - Isabel Heinemann, sur les valeurs familiales dans les bourgeoisies allemande et étatsunienne au $\mathrm{XX}^{\mathrm{e}}$ siècle. De plus, dans un livre qui laisse place à de vastes développements sur des objets aussi peut courants que l'histoire sémantique du terme de "bourgeois/citoyen » en Chine ou au Japon - ce dont il faut se réjouir - ou qui, plus généralement, entend prendre en charge des questions laissées de côté par les recherche des années 1980 et 1990, il est étonnant qu'aucune place n'ait été trouvée à la fois pour plus d'historiennes et pour l'histoire des femmes et du genre. Plus de trente ans après Bürgerinnen und Bürger publié sous la direction d'Ute Frevert en 1988, un bilan des travaux parus depuis sur la question aurait été particulièrement le bienvenu. Il est vrai, pourrait-on ajouter de manière quelque peu sarcastique mais à peine exagérée, que l'histoire globale (dans sa définition la plus lâche, dans le sens d'extra-européenne) étant à la pointe de la mode historiographique, replacer l'histoire et l'historiographie de la bourgeoisie " au-delà de l'Europe » (selon le titre de la partie IV) a pu sembler une nécessité plus grande aux éditeurs.

C'est ici d'ailleurs un autre regret : plutôt que d'inciter les auteurs à mener des études et des réflexions comparatives ou transnationales (Isabel Heinmann se distingue également sur ce point), l'Allemagne est au centre de l'ouvrage, et les autres pays et aires géographiques sont renvoyés à une partie spécifique ou alors pris en compte seulement dans les réflexions générales, sans qu'il s'agisse d'une étude empirique 
(C. Dejung). C'est très largement ce qui caractérisait les travaux de la fin du $\mathrm{XX}^{\mathrm{e}}$ siècle, comme le soulignent notamment Langewiesche et Mergel. L'histoire comparée ou croisée - menée de manière systématique sur des études de cas, par exemple une profession bourgeoise - des bourgeoisies européennes voire mondiales (et non pas seulement une collection d'études monographiques menées séparément sur chaque pays) reste donc encore largement un chantier à mener, chantier que les éditeurs n'ont pas semblé devoir rappeler.

Malgré ces limites, cet ouvrage constitue un apport important pour l'histoire et l'historiographie de la bourgeoisie en Allemagne. Même d'un point de vue strictement allemand ou européen, il témoigne de la richesse de cet objet, comme le montrent les articles des parties II et III. Historiens et historiennes, ont encore de nombreuses recherches à mener; peut-être la réhabilitation de l'histoire sociale sous de nouvelles formes permettrait-elle de nouvelles avancées et de nouveaux questionnements.

INDEX

Index chronologique : Époque contemporaine

Thèmes : Histoire sociale 